\title{
Constraining LDPC Degree Distributions for Improved Error Floor Performance
}

\author{
Sarah J. Johnson, Member, IEEE, and Steven R. Weller, Member, IEEE
}

\begin{abstract}
The error floor performance of finite-length irregular low-density parity-check (LDPC) codes can be very poor if code degree distributions are chosen to optimize the threshold performance. In this paper we show that by constraining the optimization process, a balance between threshold and error floor performance can be obtained. The resulting degree distributions give the best threshold performance subject to some minimum requirement on the error floor.
\end{abstract}

Index Terms-Low-density parity-check codes, error floor, density evolution.

\section{INTRODUCTION}

$\mathbf{T}$ HE Tanner graph of a binary low-density parity-check (LDPC) code is a bipartite graph with a bit node representing each codeword bit in the code and a check node representing each parity-check equation. A check node is connected to a bit node by a graph edge if the corresponding codeword bit is included in that parity-check equation. The bit node degree distribution of the code is denoted by

$$
\lambda(x)=\lambda_{2} x+\cdots+\lambda_{i} x^{i-1}+\cdots+\lambda_{\gamma_{\max }} x^{\gamma_{\max }-1},
$$

where $\lambda_{i}$ is the fraction of Tanner graph edges which emanate from degree- $i$ bit nodes. The fraction of degree- $i$ bit nodes is given by

$$
\psi_{i}=\frac{\lambda_{i} / i}{\sum_{j} \lambda_{j} / j} .
$$

The check degrees are similarly represented by

$$
\rho(x)=\rho_{2} x+\cdots+\rho_{i} x^{i-1}+\cdots+\rho_{r_{\max }} x^{r_{\max }-1} .
$$

A cycle in a Tanner graph is a sequence of connected nodes which start and end at the same node in the graph and contain no other nodes more than once. The length of the cycle is the number edges it contains and the girth of a graph is the length of its smallest cycle.

The degree distribution pair for a given rate LDPC code is typically selected by optimizing the code threshold returned by density evolution [1], a process which ignores error floor performance. In this paper we show that by constraining the optimization of the degree distributions, a tradeoff between threshold and error floor performance can be achieved. The resulting optimization process returns the degree distribution

Manuscript received June 10, 2005. The associate editor coordinating the review of this letter and approving it for publication was Prof. Marc Fossorier. Work supported by the Australian Research Council under Discovery Project Grant DP0449627.

The authors are with the School of Electrical Engineering and Computer Science, University of Newcastle, Callaghan, Australia (e-mail: \{sarah.johnson, steven.weller\}@ newcastle.edu.au).

Digital Object Identifier 10.1109/LCOMM.2006.02001. which gives the best threshold performance given some minimum requirement on the word error rate (WER) floor. We show that on the binary erasure channel (BEC) and binaryinput additive white Gaussian noise channel (BIAWGNC) an improvement in the bit error rate floor by up to four orders of magnitude can be achieved by trading off a small reduction in threshold.

\section{Constrained Degree Distributions}

The BEC has provided a useful framework for understanding the performance of finite-length LDPC codes (see e.g. [2]), and many of the observations made using the BEC can be usefully applied to more general channels.

On the BEC a transmitted symbol is either received correctly or completely erased with some probability $\epsilon$. If only one of the bits in a given parity-check equation is erased it can be determined exactly by replacing it with the bit value which satisfies the parity-check equation. Conversely, if more than one bit in the parity-check equation is erased, no correction can be made. A stopping set is defined as a set of code bit nodes with the property that every check node connected to a bit node in the stopping set is connected to at least two nodes in the set. Thus, on the BEC, a codeword can be corrected if and only if there are no stopping sets in the set of erased bits [2].

For irregular LDPC codes the contribution to the WER is dominated by the bit nodes of degree- 2 in the code Tanner graph, and so we calculate the expected number of stopping sets in the subgraph $\mathcal{T}_{2}$ induced by the degree- 2 bit nodes, ignoring the contribution from stopping sets involving higher degree nodes. Since the degree of all the bit nodes in $\mathcal{T}_{2}$ is 2 , a stopping set of size $k$ in $\mathcal{T}_{2}$ is also a cycle of size $2 k$.

The expected number of cycles of size $k$ in a length $n$ code is [3]:

$$
\mathbb{E}_{k-\text { cycles }}\left(C_{\lambda(x), \rho(x)}, n\right)=\frac{\left(\lambda_{2} \rho^{\prime}(1)\right)^{k}}{2 k}+O\left(n^{-1 / 3}\right),
$$

and thus the average probability that a randomly chosen size$v$ subset of the $\psi_{2} n$ degree-2 bit nodes in the ensemble $C_{\lambda(x), \rho(x)}$ is a stopping set is:

$$
P_{\mathrm{SS}}\left(C_{\lambda(x), \rho(x)}, v\right)=\frac{\left(\lambda_{2} \rho^{\prime}(1)\right)^{v} / 2 v+O\left(n^{-1 / 3}\right)}{\left(\begin{array}{c}
\psi_{2} n \\
v
\end{array}\right)} .
$$

We lower bound the word error rate on the BEC with erasure probability $\epsilon$ by summing over the contribution of stopping 
sets of size $s=2, \ldots, \psi_{2} n$ in the $\psi_{2} n$ degree-2 bit nodes:

$$
\begin{array}{r}
\mathbb{E}_{\mathrm{WER}}\left(C_{\lambda(x), \rho(x)}, n, \epsilon\right) \geq \sum_{s=2}^{\psi_{2} n}\left(\begin{array}{c}
\psi_{2} n \\
s
\end{array}\right) \epsilon^{s} P_{\mathrm{SS}}\left(C_{\lambda(x), \rho(x)}, s\right) \\
=\sum_{s=2}^{\psi_{2} n} \epsilon^{s}\left(\frac{\left(\lambda_{2} \rho^{\prime}(1)\right)^{s}}{2 s}+O\left(n^{-1 / 3}\right)\right),
\end{array}
$$

where $\epsilon^{s}$ is the probability of an erasure of size at least $s$ occurring. For asymptotically long codes,

$$
\begin{gathered}
\lim _{n \rightarrow \infty} \mathbb{E}_{\mathrm{WER}}\left(C_{\lambda(x), \rho(x)}, n, \epsilon\right) \geq \lim _{\psi_{2} n \rightarrow \infty} \sum_{s=2}^{\psi_{2} n} \frac{\left(\lambda_{2} \rho^{\prime}(1) \epsilon\right)^{s}}{2 s} \\
=\ln \left(\frac{1}{\sqrt{1+\lambda_{2} \rho^{\prime}(1) \epsilon}}\right)-\frac{\left(\lambda_{2} \rho^{\prime}(1) \epsilon\right)}{2} .
\end{gathered}
$$

A similar expression for the bit error rate (BER) can be found by noting that a stopping set of size $s$ will cause $s$ uncorrected erasures following iterative decoding:

$$
\mathbb{E}_{\mathrm{BER}}\left(C_{\lambda(x), \rho(x)}, n, \epsilon\right) \geq \sum_{s=2}^{\psi_{2} n} \frac{s}{n} \frac{\left(\lambda_{2} \rho^{\prime}(1) \epsilon\right)^{s}}{2 s} .
$$

If we consider randomly constructed LDPC codes from the ensemble with degree distribution pair $(\lambda(x), \rho(x))$ and girth $g$, then the expected error floor performance of the ensemble will be dominated by stopping sets of size $g / 2$ and we can approximate the word error rate by $W_{g}$, where

$$
W_{g} \triangleq \frac{\left(\lambda_{2} \rho^{\prime}(1) \epsilon\right)^{\frac{g}{2}}}{g} .
$$

Thus to bound the ensemble degree distribution to obtain a word error rate below $W_{g}$ we propose to constrain the degree distribution to satisfy:

$$
\lambda_{2} \leq \frac{E}{\rho^{\prime}(1) \epsilon^{*}}, \quad \text { where } \quad E \triangleq\left(g W_{g}\right)^{2 / g},
$$

and $\epsilon^{*}$ is the threshold value returned by density evolution. Thus $\epsilon<\epsilon^{*}$ corresponds to the error floor region of the WER curve, making $\epsilon^{*}$ an ideal erasure probability at which to evaluate (5). Note that setting $E=1$ returns the stability constraint for the BEC (see e.g. [1]) and the traditional optimized degree distribution is returned.

Orlitsky et al. recently established in [4] that, provided $\epsilon$ is below a certain threshold, the asymptotic average block error probability of irregular LDPC codes is

$$
1-\sqrt{1-\lambda_{2} \rho^{\prime}(1) \epsilon}
$$

The derivation of (7) uses the fact that stopping sets of size-1 dominate the contribution to the WER of a randomly chosen unconstrained Tanner graph. In practice, however, LDPC codes are not constructed with repeated edges and so do not contain size-1 stopping sets; thus the expression in (5) is more useful for predicting the word error rate floor of practical codes, as will be demonstrated in Section III.

On the BIAWGNC the factors affecting sum-product decoding performance are less straightforward to determine, and we consequently model the performance of sum-product

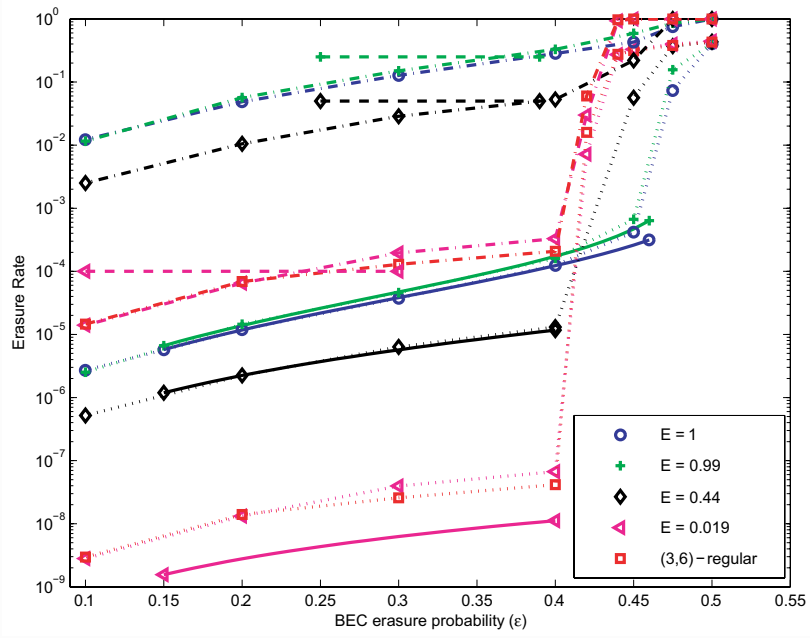

Fig. 1. The ensemble average performance on the BEC of length-10,000 rate- $\frac{1}{2}$ LDPC code ensembles without repeated edges, for degree distributions from Table I. Shown is the word erasure rate (-.-), bit erasure rate $(\cdots), \mathrm{W}_{4}$ $(--)$, and the BER bound from (4) (-).

decoding in the low noise region by that of maximumlikelihood decoding, which we can bound using the code's minimum distance distribution and the union bound. As for the BEC we restrict our attention to degree-2 nodes in the Tanner graph and note that a codeword of weight $d$ among degree-2 bit nodes is also a cycle of size $2 d$. Using this approach Di et al. [3] have shown that the expected number of codewords of weight $k$ in a length $n$ LDPC code with degree distribution pair $(\lambda(x), \rho(x))$ is also given by (1).

Using the union bound for the BIAWGNC with variance $\sigma^{2}$, we have that the approximate WER contribution of errors within bits corresponding to degree-2 nodes is

$$
\sum_{d=2}^{\psi_{2} n} \frac{\left(\lambda_{2} \rho^{\prime}(1)\right)^{d}}{2 d} Q\left(\sqrt{\frac{d}{\sigma^{2}}}\right),
$$

where

$$
Q(x)=\frac{1}{\sqrt{2 \pi}} \int_{x}^{\infty} e^{-t^{2} / 2} d t \leq \frac{1}{2} e^{-x^{2} / 2} .
$$

Considering only the impact of codewords with weight $g / 2$ in graphs with girth $g$, we define $W_{g}$ for the BIAWGNC by:

$$
W_{g} \triangleq\left(e^{\left(\frac{-1}{2 \sigma^{2}}\right)}\right)^{g / 2} \frac{\left(\lambda_{2} \rho^{\prime}(1)\right)^{g / 2}}{g},
$$

and apply the constraint:

$$
\lambda_{2} \leq \frac{E}{\rho^{\prime}(1)} e^{1 / 2 \sigma^{2}}, \quad \text { where } \quad E \triangleq\left(g W_{g}\right)^{2 / g} .
$$

Setting $E=1$ returns the stability constraint for the BIAWGNC (see e.g. [1]) and the traditional optimized degree distribution is returned.

\section{RESULTS AND DISCUSSION}

The constraints in (6) and (10) can be implemented quite simply by scaling the relevant density evolution stability condition with the required value of $E$, where $E$ is a function of both the desired constraint on the WER floor and the girth of the code to be constructed. Table I gives degree distribution pairs found in this way for the BEC and BIAWGNC, 
TABLE I

LDPC DEGREE DISTRIBUTIONS

\begin{tabular}{|c|c|c|c|c|c|c|c|c|c|c|}
\hline & \multicolumn{4}{|c|}{ Rate- $\frac{1}{2}$ (BEC) } & \multicolumn{3}{|c|}{ Rate- $\frac{1}{4}$ (BIAWGNC) } & \multicolumn{3}{|c|}{ Rate- $\frac{1}{2}$ (BIAWGNC) } \\
\hline & $\mathrm{E}=1$ & $E=0.99$ & $\mathrm{E}=0.44$ & $E=0.02$ & $\mathrm{E}=1$ & $E=0.19$ & $\mathrm{E}=0.02$ & $\mathrm{E}=1$ & $E=0.19$ & $E=0.02$ \\
\hline $\begin{array}{l}\mathrm{W}_{4} \\
\mathrm{~W}_{6}\end{array}$ & 0.25 & 0.2475 & 0.05 & $1 \times 10^{-4}$ & 0.167 & 0.00114 & $1.31 \times 10^{-6}$ & 0.25 & 0.009 & $1 \times 10^{-4}$ \\
\hline $\begin{array}{l}\lambda_{2} \\
\lambda_{3} \\
\lambda_{4} \\
\lambda_{5} \\
\lambda_{6} \\
\lambda_{7} \\
\lambda_{9} \\
\lambda_{10} \\
\end{array}$ & $\begin{array}{l}0.3371 \\
0.2653 \\
0.0237\end{array}$ & $\begin{array}{l}0.3496 \\
0.2093 \\
0.0192\end{array}$ & $\begin{array}{l}0.1689 \\
0.5690 \\
0.0009\end{array}$ & $\begin{array}{l}0.0066 \\
0.8490 \\
0.0033\end{array}$ & $\begin{array}{l}0.4310 \\
0.2203 \\
0.0035 \\
\\
0.0324 \\
0.1587 \\
\\
0.1541 \\
\end{array}$ & $\begin{array}{l}0.0872 \\
0.8650 \\
0.0242 \\
\\
0.0032 \\
0.0027 \\
\\
0.0177 \\
\end{array}$ & $\begin{array}{l}0.0086 \\
0.9711 \\
0.0006 \\
\\
0.0059 \\
0.0110 \\
\\
0.0028\end{array}$ & $\begin{array}{l}0.3282 \\
0.1581 \\
0.2275\end{array}$ & $\begin{array}{l}0.2068 \\
0.6808 \\
0.1025\end{array}$ & $\begin{array}{l}0.0082 \\
0.9689 \\
0.0077\end{array}$ \\
\hline $\begin{array}{l}\rho_{3} \\
\rho_{4} \\
\rho_{5} \\
\rho_{6} \\
\rho_{7} \\
\end{array}$ & $\begin{array}{l}0.1299 \\
0.0820 \\
0.7881 \\
\end{array}$ & $\begin{array}{l}0.0358 \\
0.1357 \\
0.8285 \\
\end{array}$ & $\begin{array}{l}0.0071 \\
0.3535 \\
0.6394 \\
\end{array}$ & $\begin{array}{l}0.0274 \\
0.2930 \\
0.6796 \\
\end{array}$ & $\begin{array}{l}0.0005 \\
0.9983 \\
0.0012\end{array}$ & $\begin{array}{l}0.0808 \\
0.8945 \\
0.0247\end{array}$ & $\begin{array}{l}0.0118 \\
0.9332 \\
0.0550\end{array}$ & $\begin{array}{l}0.02448 \\
0.35640 \\
0.61911 \\
\end{array}$ & $\begin{array}{l}0.43217 \\
0.46110 \\
0.10673 \\
\end{array}$ & $\begin{array}{l}0.35472 \\
0.09275 \\
0.55252 \\
\end{array}$ \\
\hline$* / \sigma^{*}$ & 0.4837 & 0.4820 & 0.4591 & 0.4341 & 1.39 & 1.299 & 1.269 & 0.8966 & 0.8612 & 0.8419 \\
\hline WER from (7) & 0.7220 & 0.8397 & 0.2495 & 0.0080 & & & & & & \\
\hline
\end{tabular}

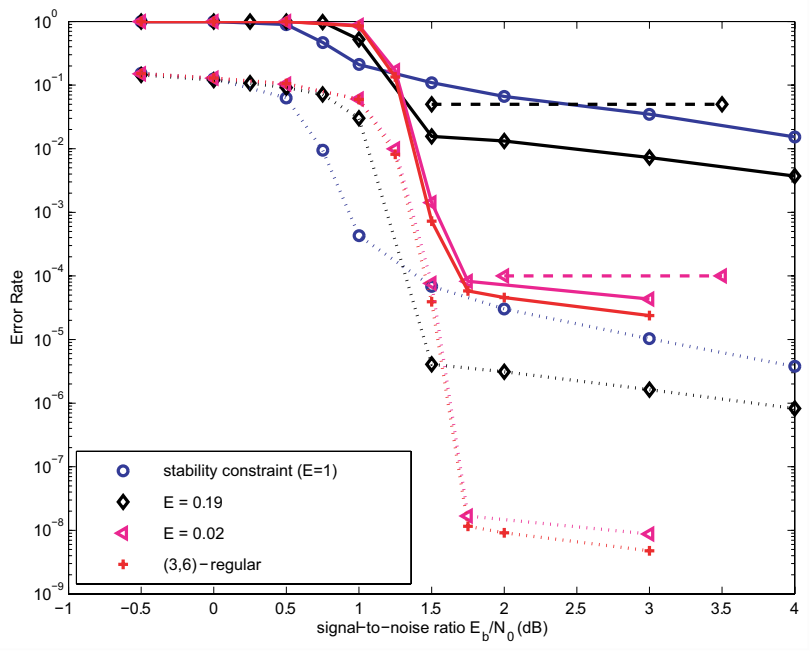

Fig. 2. The ensemble average performance on the BIAWGNC of length10,000 rate- $\frac{1}{2}$ LDPC codes with degree distributions from Table I. Shown is the word error rate (-) bit error rate $(\cdots)$ and $\mathrm{W}_{4}(---)$.

respectively. We have used differential evolution, as described in [5], to find the optimal degree distributions; thresholds for the BIAWGNC are calculated using a Gaussian approximation for density evolution from [6].

Figs. 1 to 3 show the simulated ensemble average performance, using sum-product decoding, of codes with each of these degree distributions. We see that the approximations for WER, on both the BEC and BIAWGNC, are sufficiently accurate that the proposed constraints in (6) and (10) produce the desired effect, allowing a precise tradeoff between the threshold and error floor performance to be made. By constraining the degree-2 variable nodes an error floor improvement of up to four orders of magnitude is possible. Further improvements in error floor performance may be possible if the WER bounds can be generalized so that the fraction of variable nodes with degree greater than two are also constrained.

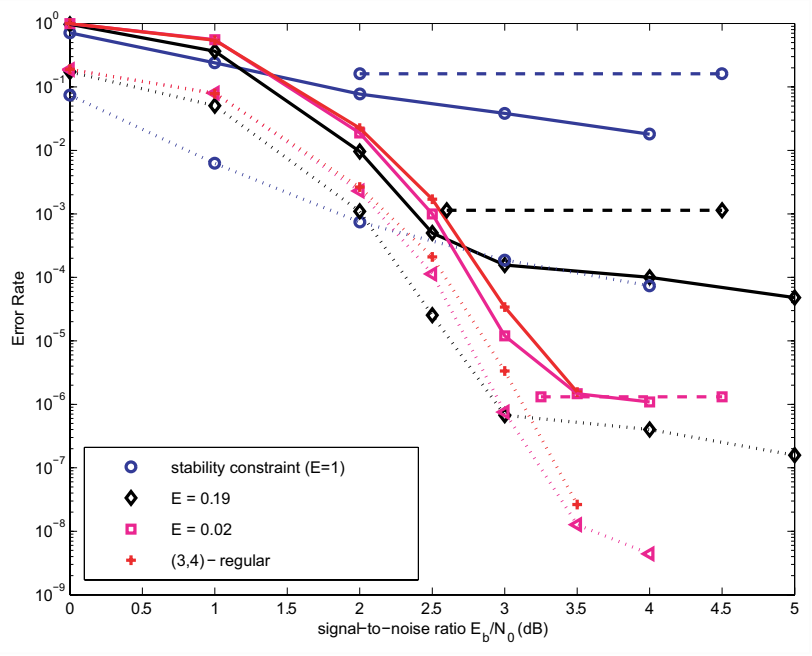

Fig. 3. The ensemble average performance of girth $\geq 6$ length-1000 rate- $\frac{1}{4}$ LDPC codes with degree distributions from Table I. Shown is the word error rate $(-)$ bit error rate $(\cdots)$ and $\mathrm{W}_{6}(---)$.

\section{REFERENCES}

[1] T. J. Richardson, M. A. Shokrollahi, and R. L. Urbanke, "Design of capacity-approaching irregular low-density parity-check codes," IEEE Trans. Inform. Theory, vol. 47, pp. 619-637, Feb. 2001.

[2] C. Di, D. Proietti, I. E. Telatar, T. J. Richardson, and R. L. Urbanke, "Finite-length analysis of low-density parity-check codes on the binary erasure channel," IEEE Trans. Inform. Theory, vol. 48, pp. 1570-1579, June 2002.

[3] C. Di, T. J. Richardson, and R. L. Urbanke, "Weight distributions of low-density parity-check codes," unpublished manuscript, available at $\langle$ http://lthcwww.epfl.ch/papers $\rangle$.

[4] A. Orlitsky, K. Viswanathan, and J. Zhang, "Stopping set distribution of LDPC code ensembles," IEEE Trans. Inform. Theory, vol. 51, pp. 929953, Mar. 2005.

[5] J. Hou, P. H. Siegel, and L. B. Milstein, "Performance analysis and code optimization of low density parity-check codes on Rayleigh fading channels," IEEE J. Select. Areas Commun., vol. 19, pp. 924-934, May 2001.

[6] F. Lehmann and G. M. Maggio, "Analysis of the iterative decoding of LDPC and product codes using the Gaussian approximation," IEEE Trans. Inform. Theory, vol. 49, pp. 2993-3000, Nov. 2003. 